\title{
General Integral Representation of the Holomorphic Functions on the Analytic Subvariety
}

By

\author{
Shu Jin CheN*
}

\section{§1. Introduction}

Henkin $^{[1]}$ and Ramirez ${ }^{[2]}$ obtained an integral representation of holomorphic functions for strictly pseudoconvex domains in $\boldsymbol{C}^{n}$. Range and $\mathrm{Siu}^{[3]}$ gave a generalization of Henkin-Ramirez's formula to the domains in $\mathbb{C}^{n}$ with piecewise smooth strictly pseudoconvex boundaries Sommer ${ }^{[4]}$ proved an integral formula of Weil type for analytic polyhedra in $\boldsymbol{C}^{n}$. Sergeev and Henkin $^{[5]}$ also obtained an integral representation for the strictly pseudoconvex ployhedra. Stout ${ }^{[6]}$ and Hatziafratis ${ }^{[7]}$ have respectively proved integral formulas for strictly pseudoconvex domains in codimension-one and codimension-m complex submanifolds of $\boldsymbol{C}^{n}$. The formula which was given by Stout is valid not only for nonsingular hypersurfaces, but also for certain subvarieties which may possess sufficiently restricted singular points. Hatziafratis' work is based on the results of Stout.

In this paper we derive integral formulas which include all the above ([1]-[7]) integral formulas for holomorphic functions. The papers of Stout ${ }^{[6]}$, Hatziafratis $^{[7]}$ and the author ${ }^{[8]}$ are most relevant references to this work.

\section{§2. Definitions, Symbols and Terms}

Definition 1 (Polyhedral domain ${ }^{[8]}$ ) Let $\Omega$ be a domain of holomorphy in $C^{n}$. An open set $D \subset \subset \Omega$ is called a polyhedral domain if there is a neighbourhood $U_{\bar{D}}$ of $\bar{D}$ and holomorphic mappings:

Communicated by K. Saito, April 26, 1990. Revised September 3, 1991.

1991 Mathematics Subject classifications: 32A25

* Department of Mathematics, Xiamen University, Xiamen Fujian, China. 


$$
X_{\alpha}: U_{\bar{D}} \rightarrow C^{m_{\alpha}}, \alpha=1,2, \cdots, N, \sum_{\alpha=1}^{N} m_{\alpha} \geqslant n,
$$

and $D_{\alpha} \subset \subset C^{m_{\alpha}}, \alpha=1,2, \cdots, N$, such that

$$
D=X_{1}^{-1}\left(D_{1}\right) \bigcap \cdots \bigcap X_{N}^{-1}\left(D_{N}\right)
$$

If $P_{1}, \cdots, P_{N}$ are differentiable functions in the neighbourhoods $\theta_{1}, \cdots, \theta_{N}$ of $\partial D_{1}, \cdots, \partial D_{N}$ respectively, and

$$
D_{\alpha} \bigcap \theta_{\alpha}=\left\{z \in \theta_{\alpha}: P_{\alpha}(z)<0\right\}, \alpha=1,2, \cdots, N,
$$

then $\partial D \subseteq X_{1}^{-1}\left(\theta_{1}\right) \bigcup \cdots \cup X_{N}^{-1}\left(\theta_{N}\right)$ and a point $z \in X_{1}^{-1}(\theta) \bigcup \cdots \cup X_{N}^{-1}\left(\theta_{N}\right)$ belongs to $D$ if and only if $z \in X_{\alpha}^{-1}\left(\theta_{\alpha}\right)$ and $P\left[X_{\alpha}(z)\right]<0$ for some $\alpha: 1 \leqslant \alpha \leqslant N . \quad D$ is called a non-degenerate polyhedral domain, if we can choose the functions $X_{\alpha}$ and $P_{\alpha}$ so that

$$
d\left(P_{\alpha_{1}} \cdot X_{\alpha_{1}}\right)(z) \wedge \cdots \wedge d\left(P_{\alpha_{l}} \cdot Z_{\alpha_{1}}\right)(z) \neq 0
$$

Whenever $P_{\alpha_{1}}\left[X_{\alpha_{1}}(z)\right]=\cdots=P_{\alpha_{l}}\left[X_{\alpha_{l}}(z)\right]=0$, for all $1 \leqslant \alpha_{1}<\cdots<\alpha_{l} \cdots \leqslant N$.

In this paper we only consider non-degenerate polyhedral domains.

A nondegenerate pohyhedral domain will be called a strictly pseudoconvex polyhedron if $P_{\alpha}(\alpha=1,2, \cdots, N)$ are strictly plurisubharmonic functions; and called a holomorphic polyhedron (including Weil polyhedron) if the mapping $P_{\alpha}(\alpha=1,2, \cdots, N)$ are pluriharmonic functions (or usual harmonic functions when $n=1)$, i.e. $P_{\alpha}(\alpha=1,2, \cdots, N)$ are twice continuously differentiable and $\partial^{2} P_{\alpha} / \partial z_{j} \partial \bar{z}_{k}=0, j, k=1,2, \cdots, n$. There exist continuously differentiable support functions for the nondegenerate polyhedral domains, support functions holomorphic in $z$ for strictly pseudoconvex polyhedrons, and holomorphic support functions for the holomorphic polyhedron.

Definition 2 (Space with slits ${ }^{[9]}$ ) A compact metric space $R$ is called a slit space or a space with slit $S$ if $S$ is a nonempty closed subsed of $R$ each point of which is an accumulation point of $R-S$ and $R-S$ is homeomorphic to a topological product $X \times Y$, where $X$ is a connected $\tilde{m}$-dimensional differential manifold of class $C^{2}$, called the base space, and $Y$ is a compact set, called the side space. The homeomorphism $\varphi: X \times Y \rightarrow R-S$ is called the coordinate function.

Example. The closure $R$ of any bounded domain in a $\tilde{n}$-dimensional 
Euclidean space $E^{\bar{n}}$ can be considered a slit space with the boundary as its slit. $\quad Y$ is then a set consisting of a single point.

Definition 3. A sequence of spaces $R_{1} \supset R_{2} \supset \cdots \supset R_{k}$ is called a chain of slit spaces, if each $R_{v}$ is a slit space with $R_{v+1}$ as its slit $(v=1, \cdots, k-1)$.

Firstly, we consider the following two types of bounded domains $D \subset \Omega$ in $\boldsymbol{C}^{n}$ :

$\langle I\rangle$ Its boundary $\partial D$ consists of a chain of slit spaces, and this chain can be written as:

$$
\partial D=\sigma^{(1)} \supset \sigma^{(2)} \supset \cdots \supset \sigma^{(\beta)}=\sigma_{1}^{(\beta-1)} \supset \sigma_{1}^{(\beta-1)} \supset \cdots \supset \sigma_{k}^{(\beta-1)}
$$

where $\sigma_{v+1}^{(\beta-1)}$ is the slit of $\sigma_{\gamma}^{(\beta-1)}, \sigma^{(i+1)}$ is the slit of $\sigma^{(i)}, \sigma_{v}^{(\beta-1)}=\bigcup_{j_{1}<\cdots<j_{v}} \sigma_{j_{1} \cdots j_{v}}^{(\beta-1)}$ $\sigma_{j_{1} \cdots j_{0}}^{(\beta-1)}$ is of real dimension $2 n-\beta-v+1 ; \sigma^{(i)}=\bigcup_{k_{1}<\cdots<k_{i}} \sigma_{k_{1} \cdots k_{i}}^{(0)}$, and $\sigma_{k_{1} \cdots k_{i}}^{(0)}$ is of real dimension $2 n-i . \quad \sigma_{k}^{(\beta-1)}$ is called the distinguished boundary of $D$.

Example. The closed bicylinder $R=\left\{\left(z_{1}, z_{2}\right):\left|z_{1}\right| \leqslant 1,\left|z_{2}\right| \leqslant 1\right\}$ can be considered a space with the boundary $R_{1}=\left\{\left(z_{1}, z_{2}\right):\left|z_{1}\right|=1,\left|z_{2}\right| \leqslant 1\right.$ and $\left.\left|z_{1}\right| \leqslant 1,\left|z_{2}\right|=1\right\}$ as slit. Moreover the boundary $R_{1}$ can also be considered a space with slit $R_{2}=\left\{\left(z_{1}, z_{2}\right):\left|z_{1}\right|=1,\left|z_{2}\right|=1\right\}$.

$\langle$ II $\rangle$ Its boundary $\partial D$ consists of a chain of slit spaces, and this chain can be written as:

$$
\partial D=\sigma^{(1)} \supset \cdots \supset \sigma^{(s)} \supset \sigma^{(t)} \supset \cdots \supset \sigma^{(\eta)} \supset \sigma^{(\beta)}=\sigma_{1}^{(\beta-1)} \supset \sigma_{2}^{(\beta-1)} \supset \cdots \supset \sigma_{k}^{(\beta-1)},
$$

where $\sigma^{(\beta)}$ is slit of $\sigma^{(\eta)}$, and the dimensions of $\sigma^{(\eta)}$ may be at least one dimension greater than the dimensions of $\sigma^{(\beta)} . \sigma_{k}^{(\beta-1)}$ is also called the distinguished boundary of $D$.

Example. The closure of all invariant subspaces of the classical domain ${ }^{[9]}$ consists of a chain of slit spaces mentioned above.

Secondly, if $F_{1}, \cdots, F_{m}$ are holomorphic functions in the neighbourhood $U_{\bar{D}}$ of $\bar{D}$, and set

$$
Z\left(F_{1}, \cdots, F_{m}\right)=\left\{z \in U_{\bar{D}}: F_{1}(z)=\cdots=F_{m}(z)=0\right\}
$$

We asume that $Z\left(F_{1}, \cdots, F_{m}\right)$ meet $\partial D$ transversally. We set $\tilde{D}=Z\left(F_{1}, \cdots, F_{m}\right)$ $\bigcap D$, and consider 
$\left\langle I^{\prime}\right\rangle \quad \partial \tilde{D}=\tilde{\sigma}^{(1)} \supset \tilde{\sigma}^{(2)} \supset \cdots \supset \tilde{\sigma}^{(\beta)}=\tilde{\sigma}_{1}^{(\beta-1)} \supset \tilde{\sigma}_{2}^{(\beta-1)} \supset \cdots \supset \tilde{\sigma}_{k}^{(\beta-1)}$,

$\left\langle\mathrm{II}^{\prime}\right\rangle \quad \partial \tilde{D}=\tilde{\sigma}^{(1)} \supset \cdots \supset \tilde{\sigma}^{(s)} \supset \tilde{\sigma}^{(t)} \supset \cdots \supset \tilde{\sigma}^{(n)} \supset \tilde{\sigma}^{(\beta)}=\tilde{\sigma}_{1}^{(\beta-1)} \supset \cdots \supset \tilde{\sigma}_{k}^{(\beta-1)}$,

where $\tilde{\sigma}^{(\theta)}=Z\left(F_{1}, \cdots, F_{m}\right) \bigcap \sigma^{(\theta)}$ and $\tilde{\sigma}_{j}^{(\beta-1)}=Z\left(F_{1}, \cdots, F_{m}\right) \bigcap \sigma_{k}^{(\beta-1)}$.

When $m=0,\left\langle\mathrm{I}^{\prime}\right\rangle$ and $\left\langle\mathrm{II}^{\prime}\right\rangle$ coincide with $\langle\mathrm{I}\rangle$ and $\langle\mathrm{II}\rangle$ respectively.

According to Hefer's theorem, we have

$F_{l}(\zeta)-F_{l}(z)=\sum_{j=1}^{n}\left(\zeta_{j}-z_{j}\right) h_{l j}(\zeta, z), l=1,2, \cdots, m$, where $h_{l j}$ are holomorphic functions on a neighbourhood of $\bar{D} \times \bar{D}$.

\section{§3. Some Lemmas}

In what follows let $D$ be a nondegenerate polyhedral domain.

Lemma 1. Let $M_{1}$ be a continuously differentiable support function for $\bar{D}$, then we have

$$
\begin{aligned}
& \bar{\partial}_{\zeta} \operatorname{det}_{(n)}\left(\frac{N_{1}}{M_{1}}, h_{1}, \cdots, h_{k}, h_{k+1}, \bar{\partial}_{\zeta}\left(\frac{N_{1}}{M_{1}}\right), \cdots, \bar{\partial}_{\zeta}\left(\frac{N_{1}}{M_{1}}\right)\right) \\
= & F_{k+1}(\zeta) \operatorname{det}_{(n)}\left(\frac{N_{1}}{M_{1}}, h_{1}, \cdots, h_{k}, \bar{\partial}_{\zeta}\left(\frac{N_{1}}{M_{1}}\right), \cdots, \bar{\partial}_{\zeta}\left(\frac{N_{1}}{M_{1}}\right)\right)
\end{aligned}
$$

on $\bar{D}-Z\left(F_{k+1}\right)$.

Proof. Since $M_{1}=M_{1}(\zeta, z)=\sum_{j=1}^{n}\left(\zeta_{j}-z_{j}\right) N_{1 j}(\zeta, z)$, i.e. $\sum_{j=1}^{n}\left(\zeta_{j}-z_{j}\right) \frac{N_{1 j}}{M_{1}}=1$, we obtain $\sum_{j=1}^{n}\left(\zeta_{j}-z_{j}\right) \bar{\partial}_{\zeta}\left(N_{1 j} / M_{1}\right)=0$. Thus we have the following determinant of $(n+1) \times(n+1)$ : 


$$
\left|\begin{array}{cccccccc}
1 & 0 & \cdots & 0 & F_{k+1} & 0 & \cdots & 0 \\
\frac{N_{11}}{M_{1}} & h_{11} & \cdots & h_{k 1} & h_{k+11} & \bar{\partial}_{\zeta}\left(\frac{N_{11}}{M_{1}}\right) & \cdots & \bar{\partial}_{\zeta}\left(\frac{N_{11}}{M_{1}}\right) \\
\vdots & \vdots & & \vdots & \vdots & \vdots & \vdots \\
\frac{N_{1 n}}{M_{1}} & h_{1 n} & \cdots & h_{k n} & h_{k+11} & \bar{\partial}_{\zeta}\left(\frac{N_{1 n}}{M_{1}}\right) & \cdots & \bar{\partial}_{\zeta}\left(\frac{N_{1 n}}{M_{1}}\right)
\end{array}\right|=0
$$

on $Z\left(F_{1}, \cdots, F_{k}\right)-Z\left(F_{k+1}\right)$. Taking it into account that $F_{1}, \cdots, F_{m}$ are the holomorphic functions and $\bar{\partial}_{\zeta} \bar{\partial}_{\zeta}\left(N_{1 j} / M_{1}\right)=0$, by (2) we have

$$
\begin{aligned}
& (-1)^{k+1} F_{k+1}(\zeta) \operatorname{det}_{(n)}\left(\frac{N_{1}}{M_{1}}, h_{1}, \cdots, h_{k}, \bar{\partial}_{\zeta}\left(\frac{N_{1}}{M_{1}}\right), \cdots, \bar{\partial}_{\zeta}\left(\frac{N_{1}}{M_{1}}\right)\right) \\
& =\operatorname{det}_{(n)}\left(h_{1}, \cdots, h_{k}, h_{k+1}, \bar{\partial}_{\zeta}\left(\frac{N_{1}}{M_{1}}\right), \cdots, \bar{\partial}_{\zeta}\left(\frac{N_{1}}{M_{1}}\right)\right) \\
& =(-1)^{k+1} \operatorname{det}_{(n)}\left(\bar{\partial}_{\zeta}\left(\frac{N_{1}}{M_{1}}\right), h_{1}, \cdots, h_{k+1}, \bar{\partial}_{\zeta}\left(\frac{N_{1}}{M_{1}}\right), \cdots, \bar{\partial}_{\zeta}\left(\frac{N_{1}}{M_{1}}\right)\right) \\
& =(-1)^{k+1} \bar{\partial}_{\zeta} \operatorname{det}_{(n)}\left(\frac{N_{1}}{M_{1}}, h_{1}, \cdots, h_{k+1}, \bar{\partial}_{\zeta}\left(\frac{N_{1}}{M_{1}}\right), \cdots, \bar{\partial}_{\zeta}\left(\frac{N_{1}}{M_{1}}\right)\right) .
\end{aligned}
$$

Thus we obtain (1).

Since

$$
\bar{\partial}_{\zeta}\left(\frac{N_{1 j}}{M_{1}}\right)=\frac{\bar{\partial}_{\zeta} N_{1 j}}{M_{1}}-\frac{N_{1 j}}{M_{1}} \cdot \frac{\bar{\partial}_{\zeta} M_{1}}{M_{1}}
$$

we can apply the properties of the determinant and write (1) as:

$$
\begin{aligned}
& \bar{\partial}_{\zeta} \frac{1}{M_{1}^{n-k-1}} \operatorname{det}_{(n)}\left(N_{1}, h_{1}, \cdots, h_{k}, h_{k+1}, \bar{\partial}_{\zeta} N_{1}, \cdots, \bar{\partial}_{\zeta} N_{1}\right) \\
& =\frac{F_{k+1}(\zeta)}{M_{1}^{n-k}} \operatorname{det}_{(n)}\left(N_{1}, h_{1}, \cdots, h_{k}, \bar{\partial}_{\zeta} N_{1}, \cdots, \bar{\partial}_{\zeta} N_{1}\right) .
\end{aligned}
$$

Especially when $k=0$, we have 


$$
\bar{\partial}_{\zeta} \operatorname{det}_{(n)}\left(\frac{N_{1}}{M_{1}}, h_{1}, \frac{\bar{\partial}_{\zeta} N_{1}}{M_{1}}, \cdots, \frac{\bar{\partial}_{\zeta} N_{1}}{M_{1}}\right)=\frac{F_{1}(\zeta)}{M_{1}^{n}} \operatorname{det}_{(n)}\left(N_{1}, \bar{\partial}_{\zeta} N_{1}, \cdots, \bar{\partial}_{\zeta} N_{1}\right)
$$

on $\bar{D}-Z\left(F_{1}\right)$.

\section{Lemma 2. If}

$$
\begin{aligned}
B_{k+1}^{F}(\zeta) & =\frac{(n-k-1) !}{\left|\nabla_{k+1}^{F}(\zeta)\right|^{2}} \sum_{1 \leqslant j_{1}<\cdots<j_{k+1} \leqslant n} \\
& (-1)^{j_{1}+\cdots+j_{k+1}} \frac{\partial\left(\overline{\left.F_{1}, \cdots, F_{k+1}\right)}\right.}{\partial\left(\zeta_{j_{1}}, \cdots, \zeta_{j_{k+1}}\right)} \wedge_{l \neq j_{1}, \cdots, j_{k+1}}^{\wedge} d \zeta_{l},
\end{aligned}
$$

where

$$
\left|\nabla_{k+1}^{F}(\zeta)\right|^{2}=\sum_{1 \leqslant j_{1}<\cdots<j_{k+1} \leqslant n} \frac{\partial\left(F_{1}, \cdots, F_{k+1}\right)}{\partial\left(\zeta_{j_{1}}, \cdots, \zeta_{j_{k+1}}\right)}{ }^{2} \neq 0
$$

(especially $\left.B_{0}^{F}(\zeta)=n ! \omega(\zeta), \omega(\zeta)=d \zeta_{1} \wedge \cdots \wedge d \zeta_{n}\right)$, then we have

$$
B_{k+1}^{F}(\zeta) \wedge d F_{k+1}=\frac{(-1)^{n+k}}{n-k} B_{k}^{F}(\zeta)
$$

on $D$.

$$
\begin{aligned}
\text { Remark. When } k=0 \text {, as }\left|\nabla_{1}^{F}(\zeta)\right|^{2}=\sum_{j=1}^{n}\left|\frac{\partial F_{1}}{\partial \zeta_{j}}\right|^{2} \text {, so (5) may be written as } \\
\begin{aligned}
B_{1}^{F}(\zeta) \wedge d F_{1} & =\frac{(n-1) !}{\left|\nabla_{1}^{F}(\zeta)\right|^{2}} \sum_{j=1}^{n}(-1)^{j} \frac{\overline{\partial F_{1}}}{\partial \zeta_{j}} d \zeta_{1} \wedge \cdots \wedge\left[d \zeta_{j}\right] \wedge \cdots \wedge d \zeta_{n} \wedge \\
& \sum_{j=1}^{n} \frac{\partial F_{1}}{\partial \zeta_{j}} d \zeta_{j} \\
& =(-1)^{n}(n-1) ! d \zeta_{1} \wedge \cdots \wedge d \zeta_{n}=\frac{(-1)^{n}}{n} B_{0}^{F}(\zeta) .
\end{aligned}
\end{aligned}
$$

The proof of Lemma $2^{[7]}$. First of all, Notice that $\left|\nabla_{k+1}^{F}(\zeta)\right| \neq 0$ implies $\left|\nabla_{k}^{F}(\zeta)\right| \neq 0$. Since we can assume that 


$$
\frac{\partial\left(F_{1}, \cdots, F_{k}\right)}{\partial\left(\zeta_{n-k+1}, \cdots, \zeta_{n}\right)}\left(\zeta_{0}\right) \neq 0
$$

where $\zeta_{0} \in Z\left(F_{1}, \cdots, F_{k}\right)$, according to the implicit function theorem, restricted to $Z\left(F_{1}, \cdots, F_{k}\right)$ locally at a point $\zeta_{0}$, we have

$$
\zeta_{n-k+j}=\widetilde{F}_{j}\left(\zeta^{(k)}\right), j=1, \cdots, k, \zeta^{(k)}=\left(\zeta_{1}, \cdots, \zeta_{n-k}\right)
$$

such that

$$
F_{j}\left(\zeta^{(k)}, \tilde{F}_{1}\left(\zeta^{(k)}\right), \cdots, \tilde{F}_{k}\left(\zeta^{(k)}\right)\right) \equiv 0, j=1, \cdots, k
$$

therefore, the following equations are true,

(*) $\quad \frac{\partial F_{j}}{\partial \zeta_{n-k+1}} \cdot \frac{\partial \widetilde{F}_{1}}{\partial \zeta_{i}}+\cdots+\frac{\partial F_{j}}{\partial \zeta_{n}} \cdot \frac{\partial \widetilde{F}_{k}}{\partial \zeta_{i}}=-\frac{\partial F_{j}}{\partial \zeta_{i}}, i=1, \cdots, n-k$

For a fixed sequence $1 \leqslant j_{1}<\cdots<j_{k} \leqslant n$, let us assume that

$$
1 \leqslant j_{1}<\cdots<j_{l} \leqslant n-k<n-k+1 \leqslant j_{l+1}<\cdots<j_{k} \leqslant n .
$$

Then it follows from $(*)$ that

$$
\frac{\partial\left(\widetilde{F}_{1}, \cdots, \hat{j}_{l+1}, \cdots, \hat{j}_{k}, \cdots, \widetilde{F}_{k}\right)}{\partial\left(\zeta_{j_{1}}, \cdots, \zeta_{j_{l}}\right)}=(-1)^{l} \delta_{1} \frac{\partial\left(F_{1}, \cdots, F_{k}\right)}{\partial\left(\zeta_{j_{1}}, \cdots, \zeta_{j_{k}}\right)}\left(\frac{\partial\left(F_{1}, \cdots, F_{k}\right)}{\partial\left(\zeta_{n-k+1}, \cdots, \zeta_{n}\right)}\right)^{-1},
$$

where $\delta_{1}=(-1)^{n(k-l)}(-1)^{j_{l+1}+\cdots+j_{k}}(-1)^{(k-l)(k-l-1) / 2}$.

So we have

$$
\begin{aligned}
\bigwedge_{\substack{i=1 \\
i \neq j_{1}, \cdots, j_{k}}}^{n} d \zeta_{i} & =d \zeta_{1} \wedge \cdots \hat{j}_{1} \cdots \hat{j}_{l} \cdots \wedge d \zeta_{n-k} \wedge d \zeta_{n-k+1} \wedge \cdots \hat{j}_{l+1} \cdots \hat{j}_{k} \cdots \wedge d \zeta_{n} \\
& =\delta_{2} \frac{\partial\left(\widetilde{F}_{1}, \cdots, \hat{j}_{l+1}, \cdots, \hat{j}_{k}, \cdots, \widetilde{F}_{k}\right)}{\partial\left(\zeta_{j_{1}}, \cdots, \zeta_{j_{l}}\right)} \bigwedge_{i=1}^{\wedge-k} d \zeta_{i}
\end{aligned}
$$

where $\delta_{2}=(-1)^{(k-n) l}(-1)^{j_{1}+\cdots+j_{1}}(-1)^{l(l-1) / 2}$.

From the above we obtain 


$$
\begin{aligned}
(* *) \quad(-1)^{j_{1}+\cdots+j_{k}} & \bigwedge_{\substack{i=1 \\
i \neq j_{1}, \cdots, j_{k}}}^{n} d \zeta_{i} \\
= & \delta \frac{\partial\left(F_{1}, \cdots, F_{k}\right)}{\partial\left(\zeta_{j_{1}}, \cdots, \zeta_{j_{k}}\right)}\left(\frac{\partial\left(F_{1}, \cdots, F_{k}\right)}{\partial\left(\zeta_{n-k+1}, \cdots, \zeta_{n}\right)}\right)^{-1} \bigwedge_{i=1}^{n-k} d \zeta_{i},
\end{aligned}
$$

where $\delta=(-1)^{k(n-k)}(-1)^{k(k+1) / 2}$.

It follows from $(* *)$ that

$$
\begin{aligned}
(* * *) \quad B_{k}^{F}(\zeta)= & \frac{(n-k) \delta}{\left|\nabla_{k}^{F}(\zeta)\right|^{2}} \sum_{j \leqslant j_{1}<\cdots j_{k} \leqslant n} \frac{\overline{\partial\left(F_{1}, \cdots, F_{k}\right)}}{\partial\left(\zeta_{j_{1}}, \cdots, \zeta_{j_{k}}\right)} \cdot \frac{\partial\left(F_{1}, \cdots, F_{k}\right)}{\partial\left(\zeta_{j_{1}}, \cdots, \zeta_{j_{k}}\right)} \\
& \left(\frac{\partial\left(F_{1}, \cdots, F_{k}\right)}{\partial\left(\zeta_{n-k+1}, \cdots, \zeta_{n}\right)}\right)^{-1} \bigwedge_{i=1}^{n-k} d \zeta_{i} \\
= & \delta(n-k) !\left(\frac{\partial\left(F_{1}, \cdots, F_{k}\right)}{\partial\left(\zeta_{n-k+1}, \cdots, \zeta_{n}\right)}\right)^{-1} \bigwedge_{i=1}^{n-k} d \zeta_{i} .
\end{aligned}
$$

Using the above expression, we obtain

$$
\begin{aligned}
& B_{k+1}^{F} \wedge d F_{k+1}=\frac{(n-k-1) !(-1)^{n+k}}{\left|\nabla_{k+1}^{F}(\zeta)\right|^{2}} \sum_{1 \leqslant j_{1}<\cdots<j_{k} \leqslant n}(-1)^{j_{1}+\cdots+j_{k}} \\
& {\left[\sum_{i=1}^{n} \frac{\partial F_{k+1}}{\partial \zeta_{i}} \frac{\overline{\partial\left(F_{1}, \cdots, F_{k}, F_{k+1}\right)}}{\partial\left(\zeta_{j_{1}}, \cdots, \zeta_{j_{k}}, \zeta_{i}\right)}\right] \stackrel{\substack{i=1 \\
i \neq j_{1}, \cdots, j_{k}}}{\wedge} d \zeta_{i}} \\
& =\frac{(n-k-1) !(-1)^{n+k} \delta}{\left|\nabla_{k+1}^{F}(\zeta)\right|^{2}} \sum_{j \leqslant j_{1}<\cdots j_{k} \leqslant n} \sum_{i=1}^{n} \frac{\partial F_{k+1}}{\partial \zeta_{i}} \frac{\overline{\partial\left(F_{1}, \cdots, F_{k}, F_{k+1}\right)}}{\left.\partial\left(\zeta_{i_{1}}, \cdots, \zeta_{i_{k}}, \zeta_{i}\right)\right)} . \\
& \frac{\partial\left(F_{1}, \cdots, F_{k}\right)}{\partial\left(\zeta_{j_{1}}, \cdots, \zeta_{j_{k}}\right)}\left(\frac{\partial\left(F_{1}, \cdots, F_{k}\right)}{\partial\left(\zeta_{n-k+1}, \cdots, \zeta_{n}\right)}\right)^{-1} \bigwedge_{i=1}^{n-k} d \zeta_{i} \\
& =\frac{(-1)^{n+k} \delta(n-k) !}{n-k}\left(\frac{\partial\left(F_{1}, \cdots, F_{k}\right)}{\partial\left(\zeta_{n-k+1}, \cdots, \zeta_{n}\right)}\right)^{-1} \bigwedge_{i=1}^{n-k} d \zeta_{i}=\frac{(-1)^{n+k}}{n-k} B_{k}^{F}(\zeta) .
\end{aligned}
$$

Lemma 3. Let $D_{k}=Z\left(F_{1}, \cdots, F_{k}\right) \bigcap D, \quad D_{0}=D, D_{m}=\tilde{D}$ and $M_{1}$ a continuously differentiable support function. If $f(z)$ is a holomorphic function on $\bar{D}_{k}$ and $\left|\nabla_{k+1}^{F}(\zeta)\right| \neq 0$, then 


$$
\begin{gathered}
c_{k+1} \int_{\partial D_{k+1}} \frac{f(\zeta)}{M_{1}^{n-k+1}} \operatorname{det}_{(n)}\left(N_{1}, h_{1}, \cdots, h_{k+1}, \bar{\partial}_{\zeta} N_{1}, \cdots, \bar{\partial}_{\zeta} N_{1}\right) \wedge B_{k+1}^{F}(\zeta) \\
\quad=c_{k} \int_{\partial D_{k}} \frac{f(\zeta)}{M_{1}^{n-k}} \operatorname{det}_{(n)}\left(N_{1}, h_{1}, \cdots, h_{k}, \bar{\partial}_{\zeta} N_{1}, \cdots, \bar{\partial}_{\zeta} N_{1}\right) \wedge B_{k}^{F}(\zeta),
\end{gathered}
$$

where $\quad c_{k}=(-1)^{k(n+1)}(-1)^{k(k+1) / 2} /(n-k) !(2 \pi i)^{n-k}$.

Proof. Let $\left(\partial D_{k}\right)_{\varepsilon}=\left\{\zeta \in \partial D_{k}:\left|F_{k+1}(\zeta)\right|>\varepsilon\right\} . \quad$ By lemma 1 and lemma 2, taking account that $c_{k+1}=(-1)^{n+k}(n-k) 2 \pi i c_{k}$, we obtain

$$
\begin{aligned}
& c_{k} \int_{\left(\partial D_{k}\right)_{\varepsilon}} \frac{f(\zeta)}{M_{1}^{n-k}} \operatorname{det}_{(n)}\left(N_{1}, h_{1}, \cdots, h_{k}, \bar{\partial}_{\zeta} N_{1}, \cdots, \bar{\partial}_{\zeta} N_{1}\right) \wedge B_{k}^{F}(\zeta) \\
= & c_{k} \int_{\left(\partial D_{k}\right)_{\varepsilon}} d_{\zeta}\left[\frac{f(\zeta)}{M_{1}^{n-k-1}} \operatorname{det}_{(n)}\left(N_{1}, h_{1}, \cdots, h_{k}, h_{k+1}, \bar{\partial}_{\zeta} N_{1}, \cdots, \bar{\partial}_{\zeta} N_{1}\right)\right. \\
& \left.\wedge(-1)^{n+k}(n-k) B_{k+1}^{F}(\zeta) \wedge \frac{d F_{k+1}}{F_{k+1}}\right] \\
= & (n-k)(-1)^{n+k} c_{k} \int_{\partial\left(\partial D_{k}\right) \varepsilon} \frac{f(\zeta)}{M_{1}^{n-k-1}} \operatorname{det}_{(n)}\left(N_{1}, h_{1}, \cdots, h_{k+1}, \bar{\partial}_{\zeta} N_{1}, \cdots, \bar{\partial}_{\zeta} N_{1}\right) \\
& \wedge B_{k+1}^{F}(\zeta) \wedge \frac{d F_{k+1}}{F_{k+1}} \\
= & \frac{c_{k+1}}{2 \pi i} \int_{|\tau|=\varepsilon}\left[\int _ { \{ \zeta \in \partial D _ { k } : F _ { k + 1 } ( \zeta ) = \tau \} } \frac { f ( \zeta ) } { M _ { 1 } ^ { n - k - 1 } } \operatorname { d e t } _ { ( n ) } \left(N_{1}, h_{1}, \cdots, h_{k+1},\right.\right. \\
& \left.\left.\bar{\partial}_{\zeta} N_{1}, \cdots, \bar{\partial}_{\zeta} N_{1}\right) \wedge B_{k+1}^{F}(\zeta)\right] \frac{d \tau}{\tau} .
\end{aligned}
$$

Let $s_{0}=\partial D_{k+1}=\left\{\zeta \in \partial D_{k}: F_{k+1}(\zeta)=0\right\}$ and $v(\zeta)$ be the normal direction at $\zeta \in s_{0}$. We consider the smooth mapping $f:(\zeta, \tau) \rightarrow \zeta+\tau \nu(\zeta), s_{0} \times\{|\tau|<\varepsilon\} \rightarrow$ $\left.V_{0} \equiv\left\{\zeta \in \partial D_{k}: F_{k+1}(\zeta)=\tau\right\}\right|_{|\tau|<\epsilon}$. Since $s_{0}$ is compact and the Jacobian $\left.J f\right|_{(\zeta, 0)} \neq 0$ for every $(\zeta, 0)$ in $\mathbb{R}^{2 n-2 k-1}$, there is the inverse $f^{-1}$. Here $\varepsilon$ is chosen to be sufficiently small. From the above, we conclude that $\left\{\zeta \in \partial D_{k}: F_{k+1}(\zeta)=\tau\right\}$ is (for $\tau \in C,|\tau|<\varepsilon$ and $\varepsilon$ a small positive number) diffeomorphic to $\partial D_{k+1}$.

When $\varepsilon \rightarrow 0$, the left side and right side of (7) tend to the right side 
and left side of (6) respectively.

Corollary 1 . Let $f(z)$ be a holomorphic function on $\bar{D}_{1}$, then when $z \in D_{1}$, and $\left|\nabla_{1}^{F}(\zeta)\right| \neq 0$ on $\partial D_{1}$, we have

$$
f(z)=c_{1} \int_{\partial D_{1}} \frac{f(\zeta)}{M_{1}^{n-1}} \operatorname{det}_{(n)}\left(N_{1}, h_{1}, \bar{\partial}_{\zeta} N_{1}, \cdots, \bar{\partial}_{\zeta} N_{1}\right) \wedge B_{1}^{F}(\zeta)
$$

Proof. Applying remark for lemma 2 and (6), we have

$$
\begin{aligned}
& c_{1} \int_{\partial D_{1}} \frac{f(\zeta)}{M_{1}^{n-1}} \operatorname{det}_{(n)}\left(N_{1}, h_{1}, \bar{\partial}_{\zeta} N_{1}, \cdots, \bar{\partial}_{\zeta} N_{1}\right) \wedge B_{1}^{F}(\zeta) \\
& =c_{0} \int_{\partial D_{0}} \frac{f(\zeta)}{M_{1}^{n}} \operatorname{det}_{(n)}\left(N_{1}, \bar{\partial}_{\zeta} N_{1}, \cdots, \bar{\partial}_{\zeta} N_{1}\right) \wedge n ! \omega(\zeta) \\
& =\frac{1}{(2 \pi i)^{n}} \int_{\partial D} \frac{f(\zeta)}{M_{1}^{n}} \operatorname{det}_{(n)}\left(N_{1}, \bar{\partial}_{\zeta} N_{1}, \cdots, \bar{\partial}_{\zeta} N_{1}\right) \wedge \omega(\zeta) .
\end{aligned}
$$

By Canchy-Fantappie formula, the right-hand side of (9) equals to $f(z)$, and (8) is obtained.

Remark. In fact, representation (8) is more evident than that in [6].

Corollary 2. Let $f(z)$ be a holomorphic function on $\overline{\tilde{D}}$, then when $z \in \tilde{D}$ and $\left|\nabla_{m}^{F}(\zeta)\right| \neq 0$ on $\partial \tilde{D}$, we have

$$
f(z)=c_{m} \int_{\partial \tilde{D}} \frac{f(\zeta)}{M_{1}^{n-m}} \operatorname{det}_{(n)}\left(N_{1}, h_{1}, \cdots, h_{m}, \bar{\partial}_{\zeta} N_{1}, \cdots, \bar{\partial}_{\zeta} N_{1}\right) \wedge B_{m}^{F}(\zeta)
$$

Proof. By lemma 3 and its corollary 1, we obtain (10).

Lemma 4. Let $T_{0}^{(i)}=\sum_{j=1}^{n}\left(\zeta_{j}-z_{j}\right) S_{0 j}^{(i)}(\zeta, z) \neq 0(\zeta \neq z)$ for some continuous functions $S_{0 j}^{(i)}(i=1,2)$ on $D$, and let $T_{l}=\sum_{j=1}^{n}\left(\zeta_{j}-z_{j}\right) s_{l j}(\zeta, z)(l=1,2, \cdots, n-k-1)$ be continuously differentiable support functions for $\bar{D}$. Then we have

$$
\operatorname{det}_{(n)}\left(\frac{S_{0}^{(1)}}{T_{0}^{(1)}}, h_{1}, \cdots, h_{k}, \bar{\partial}_{\zeta}\left(\frac{S_{1}}{T_{1}}\right), \cdots, \bar{\partial}_{\zeta}\left(\frac{S_{n-k-1}}{T_{n-k-1}}\right)\right)
$$




$$
=\operatorname{det}_{(n)}\left(\frac{S_{0}^{(2)}}{T_{0}^{(2)}}, h_{1}, \cdots, h_{k}, \bar{\partial}_{\zeta}\left(\frac{S_{1}}{T_{1}}\right), \cdots, \bar{\partial}_{\zeta}\left(\frac{S_{n-k-1}}{T_{n-k-1}}\right)\right)
$$

on $D_{k}$.

Proof. Since $\sum_{j=1}^{n}\left(\zeta_{j}-z_{j}\right) S_{l j} / T_{l}=1$, then

$$
\sum_{j=1}^{n}\left(\zeta_{j}-z_{j}\right) \bar{\partial}_{\zeta}\left(S_{l j} / T_{l}\right)=0, \quad l=1, \cdots, n-k-1
$$

Since

$$
\sum_{j=1}^{n}\left(\zeta_{j}-z_{j}\right)\left(\frac{S_{0 j}^{(1)}}{T_{0}^{(1)}}-\frac{S_{0 j}^{(2)}}{T_{0}^{(2)}}\right)=0
$$

and on $D_{k}$

$$
0=F_{l}(\zeta)-F_{l}(z)=\sum_{j=1}^{n}\left(\zeta_{j}-z_{j}\right) h_{l j}(\zeta, z), l=1, \cdots, k
$$

thus by (12)-(14), we obtain

$$
\operatorname{det}_{(n)}\left(\frac{S_{0}^{(1)}}{T_{0}^{(1)}}-\frac{S_{0}^{(2)}}{T_{0}^{(2)}}, h_{1}, \cdots, h_{k}, \bar{\partial}_{\zeta}\left(\frac{S_{1}}{T_{1}}\right), \cdots, \bar{\partial}_{\zeta}\left(\frac{S_{n-k-1}}{T_{n-k-1}}\right)\right)=0
$$

on $D_{k}$, i.e. we have (11) on $D_{k}$.

Lemma 5. Let $T_{l}=\sum_{j=1}^{n}\left(\zeta_{j}-z_{j}\right) S_{l j}(\zeta, z), l=0,1,2, \cdots, n-k-1$, be continuous differentiable support functions for $\bar{D}$, then

$$
\begin{array}{r}
\operatorname{det}_{(n)}\left(\frac{S_{0}}{T_{0}}, h_{1}, \cdots, h_{k}, \bar{\partial}_{\zeta}\left(\frac{S_{1}}{T_{1}}\right), \cdots, \bar{\partial}_{\zeta}\left(\frac{S_{l}}{T_{l}}\right), \cdots, \bar{\partial}_{\zeta}\left(\frac{S_{n-k-1}}{T_{n-k-1}}\right)\right) \\
-\operatorname{det}_{(n)}\left(\frac{S_{0}}{T_{0}}, h_{1}, \cdots, h_{k}, \bar{\partial}_{\zeta}\left(\frac{S_{1}}{T_{1}}\right), \cdots, \bar{\partial}_{\zeta}\left(\frac{\tilde{S}_{l}}{\widetilde{T}_{l}}\right), \cdots, \bar{\partial}_{\zeta}\left(\frac{S_{n-k-1}}{T_{n-k-1}}\right)\right)
\end{array}
$$


is the exact differential form of $\bar{\partial}$ on $D_{k}$. Here $\widetilde{S}_{l}$ and $\tilde{T}_{l}$ are of the same properties as $S_{l}$ and $T_{l}$ respectively.

Proof. Since

$$
\begin{aligned}
& \bar{\partial}_{\zeta} \operatorname{det}_{(n)}\left(\frac{S_{l}}{T_{l}}, h_{1}, \cdots, h_{k}, \bar{\partial}_{\zeta}\left(\frac{S_{1}}{T_{1}}\right), \cdots, \frac{\tilde{S}_{l}}{\tilde{T}_{l}}, \cdots, \bar{\partial}_{\zeta}\left(\frac{S_{n-k-1}}{T_{n-k-1}}\right)\right) \\
& =\operatorname{det}_{(n)}\left(\bar{\partial}_{\zeta}\left(\frac{S_{l}}{T_{l}}\right), h_{1}, \cdots, h_{k}, \bar{\partial}_{\zeta}\left(\frac{S_{1}}{T_{1}}\right), \cdots, \frac{\tilde{S}_{l}}{\widetilde{T}_{l}}, \cdots, \bar{\partial}_{\zeta}\left(\frac{S_{n-k-1}}{T_{n-k-1}}\right)\right) \\
& +(-1)^{l-1} \operatorname{det}_{(n)}\left(\frac{S_{l}}{T_{l}}, h_{1}, \cdots, h_{k}, \bar{\partial}_{\zeta}\left(\frac{S_{1}}{T_{1}}\right), \cdots, \bar{\partial}_{\zeta}\left(\frac{\widetilde{S}_{l}}{\tilde{T}_{l}}\right), \cdots, \bar{\partial}_{\zeta}\left(\frac{S_{n-k-1}}{T_{n-k-1}}\right)\right) \\
& =(-1)^{l} \operatorname{det}_{(n)}\left(\frac{\tilde{S}_{l}}{\widetilde{T}_{l}}, h_{1}, \cdots, h_{k}, \bar{\partial}_{\zeta}\left(\frac{S_{1}}{T_{1}}\right), \cdots, \bar{\partial}_{\zeta}\left(\frac{S_{l}}{T_{l}}\right), \cdots, \bar{\partial}_{\zeta}\left(\frac{S_{n-k-1}}{T_{n-k-1}}\right)\right) \\
& \quad+(-1)^{l-1} \operatorname{det}_{(n)}\left(\frac{S_{l}}{T_{l}}, h_{1}, \cdots, h_{k}, \bar{\partial}_{\zeta}\left(\frac{S_{1}}{T_{1}}\right), \cdots, \bar{\partial}_{\zeta}\left(\frac{\tilde{S}_{l}}{\tilde{T}_{l}}\right), \cdots, \bar{\partial}_{\zeta}\left(\frac{S_{n-k-1}}{T_{n-k-1}}\right)\right),
\end{aligned}
$$

by lemma 4 , replacing $\widetilde{S}_{l} / \widetilde{T}_{l}$ and $S_{l} / \mathrm{T}_{l}$ by $S_{0} / \mathrm{T}_{0}$ in two determiants of the right-hand side of (16), we can conclude that (15) is a exact differential form of $\bar{\partial}$.

Corollary. With the identical assumptions of lemma 5,

$$
\begin{aligned}
& \operatorname{det}_{(n)}\left(\frac{S_{0}}{T_{0}}, h_{1}, \cdots, h_{k}, \bar{\partial}_{\zeta}\left(\frac{S_{1}}{T_{1}}\right), \cdots, \bar{\partial}_{\zeta}\left(\frac{S_{n-k-1}}{T_{n-k-1}}\right)\right) \\
& -\operatorname{det}_{(n)}\left(\frac{S_{0}}{T_{0}}, h_{1}, \cdots, h_{k}, \bar{\partial}_{\zeta}\left(\frac{\tilde{S}_{l}}{\tilde{T}_{l}}\right), \cdots, \bar{\partial}_{\zeta}\left(\frac{S_{n-k-1}}{T_{n-k-1}}\right)\right)
\end{aligned}
$$

is a exact differential form of $\bar{\partial}$. Here $\tilde{S}_{1}, \cdots, \widetilde{S}_{n-k-1}$ and $\tilde{T}_{1}, \cdots, \widetilde{T}_{n-k-1}$ are of the same properties as $S_{1}, \cdots, S_{n-k-1}$ and $T_{1}, \cdots, T_{n-k-1}$ respectively.

\section{§4. Main Theorems}

Let $\left\{U_{j}\right\}_{j=1}^{N}$ be a finite open covering of an open neighbourhood $U$ of 
$\partial D$, and let $X_{j}: U_{j} \rightarrow \boldsymbol{R}(1 \leqslant j \leqslant N)$ be $C^{1}$ functions, such that

(i) $D \bigcap U_{\partial D}=\left\{z \in U\right.$ : for $1 \leqslant j \leqslant N$ either $z \notin U_{j}$ or $\left.X_{j}(z)<0\right\}$,

(ii) for $1 \leqslant i_{1},<\cdots<i_{l} \leqslant N, d X_{i_{1}}, \cdots, d X_{i_{l}}$ are linearly independent over $\mathbb{R}$ at every point of $\bigcap_{v=1}^{l} U_{i_{v}}$.

For every ordered subset $\left\{j_{1}, \cdots, j_{\theta}\right\}$ of $\{1, \cdots, N\}$, define

$$
\sigma_{j_{1} \cdots j_{\theta}}^{(0)}=\left\{z \in \partial D \bigcap_{\alpha=1}^{\theta} U_{j_{\alpha}}: X_{j_{1}}(z)=\cdots=X_{j_{\theta}}(z)=0\right\}
$$

and choose the orientation on $\sigma_{j_{1} \cdots j_{\theta}}^{(0)}$ such that the orientation is skew symmetric in $\left(j_{1}, \cdots, j_{\theta}\right)$ and the following equations hold when $D$ is given the natural orientation:

$$
\partial D=\bigcup_{j=1}^{N} \sigma_{j}^{(0)}, \sigma^{(\theta)}=\bigcup_{j_{1}<\cdots<j_{\theta}} \sigma_{j_{1} \cdots j_{\theta}}^{(0)} \partial \sigma_{j_{1} \cdots j_{\theta}}^{(0)}=\bigcup_{j=1}^{N} \sigma_{j_{1} \cdots j_{\theta j} j}^{(0)} .
$$

$\tilde{\sigma}_{j_{1} \cdots j_{\theta}}^{(0)}, \partial \tilde{D}, \tilde{\sigma}^{(\theta)}, \partial \tilde{\sigma}_{j_{1} \cdots j_{\theta}}^{(0)}$ is defined as above, and it is easy to verify the following (cf. [3]):

$$
\partial\left(\sum_{\Theta}(-1)^{\theta} \tilde{\sigma}^{(\theta)} \times \Delta_{0}^{(\theta)}\right)=\sum_{\Theta} \tilde{\sigma}^{(\theta)} \times \Delta^{(\theta-1)}-\tilde{\sigma}^{(1)} \times \Delta_{0}^{(0)},
$$

where $\Theta=\left\{j_{1}, \cdots, j_{\theta}\right\}$ is a ordered subset of $\{1, \cdots, N\}, j_{1}<\cdots<j_{\theta}$;

$$
\begin{aligned}
& \Delta=\left\{\mu=\left(\mu_{0}, \mu_{1}, \cdots, \mu_{N}\right) \in R^{N+1}: \mu_{j} \geqslant 0, \sum_{j=0}^{N} \mu_{j}=1\right\}, \\
& \Delta_{0 j_{1} \cdots j_{\theta}}^{(\theta)}=\left\{\mu \in \Delta: \mu_{0}+\mu_{j_{1}}+\cdots+\mu_{j_{0}}=1, \Delta_{0}^{(0)}=(1,0, \cdots, 0),\right. \\
& \Delta_{0}^{(\theta)}=\bigcup_{j_{1}<\cdots<j_{\theta}} \Delta_{0 j_{1} \cdots j_{\theta}}^{(0)}, \Delta^{(\theta-1)}=\bigcup_{j_{1}<\cdots<j_{\theta}} \Delta_{j_{1} \cdots j_{\theta} \cdot}^{(\theta-1)}
\end{aligned}
$$

Theorem 1. Let $D$ be a nondegenesate polyhedral domain in $C^{n}$ whose boundary can be written as a chain of slit spaces;

$$
\sigma^{(1)} \supset \sigma^{(2)} \supset \cdots \supset \sigma^{(\beta)}
$$


Assume that $Z\left(F_{1}, \cdots, F_{m}\right)$ meets $\partial D$ transversally yielding a chain of slit spaces;

$$
\partial \widetilde{D}=\tilde{\sigma}^{(1)} \supset \tilde{\sigma}^{(2)} \supset \cdots \supset \tilde{\sigma}^{(\beta)},
$$

and that $\left|\nabla_{m}^{F}(\zeta)\right| \neq 0$ on $\partial \tilde{D}$. Then

$$
f(z)=c_{m} \sum_{\Theta} \int_{\widetilde{\sigma}^{(\theta)} \times \Delta^{(\theta-1)}} f(\zeta) \operatorname{det}_{(n)}\left(Q, h_{1}, \cdots, h_{m}, \partial_{\bar{\zeta} \mu} Q, \cdots, \partial_{\bar{\zeta} \mu} Q\right) \wedge B_{m}^{F}(\zeta) \text { if } \beta>1
$$

and

$$
f(z)=c_{m} \int_{\partial \tilde{D}} \frac{f(\zeta)}{M_{0}^{n-m}} \operatorname{det}_{(n)}\left(N^{0}, h_{1}, \cdots, h_{m}, \partial_{\bar{\zeta}} N^{0}, \cdots, \partial_{\bar{\zeta}} N^{0}\right) \wedge B_{m}^{F}(\zeta) \text { if } \beta=1,
$$

for any holomorphic function on $\overline{\bar{D}}$ and $z \in \tilde{D}$. Here

$$
Q=\left(Q_{1}, \cdots, Q_{n}\right)^{t}, Q_{p}=\mu_{0} \frac{N_{p}^{0}(\zeta, z)}{M_{0}}+\sum_{j \in \Theta} \mu_{j} \frac{N_{j p}(\zeta, z)}{M_{j}} \text { on } \Delta_{0}^{(\theta)}
$$

where $M_{0}=\sum_{p=1}^{n}\left(\zeta_{p}-z_{p}\right) N_{p}^{0}(\zeta, z) \neq 0($ when $\zeta \neq z), M_{j}=\sum_{p=1}^{n}\left(\zeta_{p}-z_{p}\right) N_{j p}(\zeta, z) \neq 0$ (when $\zeta \neq z$ ), i.e. $M_{0}, M_{j}$ are the continuously differentiable support functions; and $Q_{p}=\sum_{j \in \Theta} \mu_{j} N_{j p}(\zeta, z) / M_{j}$ on $\Delta^{(\theta-1)}$.

Proof. Since $\sum_{j=1}^{n}\left(\zeta_{j}-z_{j}\right) Q_{j}(\zeta, z, \mu) \equiv 1 \quad$ on $\quad \Delta_{0}^{(\theta)}$, then $\sum_{j=1}^{n}\left(\zeta_{j}-z_{j}\right) \partial_{\bar{\zeta} \mu}$ $Q(\zeta, z, \mu)=0$. According to Hefer's theorem, we have $0=F_{l}(\zeta)-F_{l}(z)=$ $\sum_{j=1}^{n}\left(\zeta_{j}-z_{j}\right) h_{l j}(\zeta, z)$ on $\partial \tilde{D} \times \tilde{D}, l=1, \cdots, m . \quad$ By $(* * *)$ we have $\bar{\partial}_{\zeta} B_{m}^{F}(\zeta)=0 . \quad$ As a result we obtain

$$
\begin{aligned}
& d\left[\operatorname{det}_{(n)}\left(Q, h_{1}, \cdots, h_{m}, \partial_{\bar{\zeta} \mu} Q, \cdots, \partial_{\bar{\zeta} \mu} Q\right) \wedge B_{m}^{F}(\zeta)\right] \\
& =\operatorname{det}_{(n)}\left(\partial_{\bar{\zeta} \mu} Q, h_{1}, \cdots, h_{m}, \partial_{\bar{\zeta} \mu} Q, \cdots, \partial_{\bar{\zeta} \mu} Q\right) \wedge B_{m}^{F}(\zeta)=0
\end{aligned}
$$

on $\tilde{\sigma}^{(\theta)} \times \Delta_{0}^{(\theta)}$. Using Stokes' theorem and taking account of (18) and (26), we obtain 


$$
\begin{aligned}
& c_{m} \sum_{\Theta} \int_{\tilde{\sigma}^{(\theta) \times \Delta^{(\theta-1)}}} f(\zeta) \operatorname{det}_{(n)}\left(Q, h_{1}, \cdots, h_{m}, \partial_{\bar{\zeta} \mu} Q, \cdots, \partial_{\bar{\zeta} \mu} Q\right) \wedge B_{m}^{F}(\zeta) \\
& =c_{m} \int_{\widetilde{\sigma}^{(1) \times \Delta_{0}^{(0)}}} f(\zeta) \operatorname{det}_{(n)}\left(Q, h_{1}, \cdots, h_{m}, \partial_{\bar{\zeta} \mu} Q, \cdots, \partial_{\bar{\zeta} \mu} Q\right) \wedge B_{m}^{F}(\zeta) \\
& =c_{m} \int_{\partial \tilde{D}} f(\zeta) \operatorname{det}_{(n)}\left(\frac{N^{0}}{M_{0}}, h_{1}, \cdots, h_{m}, \partial_{\bar{\zeta}}\left(\frac{N^{0}}{M_{0}}\right), \cdots, \partial_{\bar{\zeta}}\left(\frac{N^{0}}{M_{0}}\right)\right) \wedge B_{m}^{F}(\zeta) \\
& =c_{m} \int_{\partial \tilde{D}} \frac{f(\zeta)}{M_{0}^{n-m}} \operatorname{det}_{(n)}\left(N^{0}, h_{1}, \cdots, h_{m}, \partial_{\bar{\zeta}} N^{0}, \cdots, \partial_{\bar{\zeta}} N^{0}\right) \wedge B_{m}^{F}(\zeta) .
\end{aligned}
$$

Applying corollary 2 of lemma 3 to the right-hand side of (27), we obtain (24).

Remark. Obviously, (24) includes the generalizations of Range and Siu's formula ${ }^{[3]}$, and of Sergeev and Henkin's formula ${ }^{[5]}$ on the analytic subvariety.

Theorem 2. Let $f(z)$ be a holomorphic function on $\overline{\tilde{D}}$, then, for $z \in \tilde{D}$, we have

$$
f(z)=c_{m} \int_{\partial \tilde{D}} f(\zeta) \operatorname{det}_{(n)}\left(\frac{S_{0}}{T_{0}}, h_{1}, \cdots, h_{m}, \bar{\partial}_{\zeta}\left(\frac{S_{1}}{T_{1}}\right), \cdots, \bar{\partial}_{\zeta}\left(\frac{S_{n-m-1}}{T_{n-m-1}}\right)\right) \wedge B_{m}^{F}(\zeta)
$$

Remark 1. When $m=0, c_{m}=1 / n !(2 \pi i)^{n}, B_{0}^{F}(\zeta)=n ! \omega(\zeta)$ in (28), and (28) can be rewritten as:

$$
f(z)=\frac{1}{(2 \pi i)^{n}} \int_{\partial D} f(\zeta) \operatorname{det}_{(n)}\left(\frac{S_{0}}{T_{0}}, \bar{\partial}_{\zeta}\left(\frac{S_{1}}{T_{1}}\right), \cdots, \bar{\partial}_{\zeta}\left(\frac{S_{n-1}}{T_{n-1}}\right)\right) \wedge \omega(\zeta)
$$

This is the generalized Cauchy-Fantappie formula for the bounded domains in $C^{n}$. In fact, let $S_{1}=\cdots=S_{n-m-1}=S_{0}$, (29) is the Cauchy-Fantappie formula.

Remark 2. If $S_{1}=\cdots=S_{n-m-1}=S_{0}$, then $T_{1}=\cdots=T_{n-m-1}=T_{0}$. Thus (28) is the generalization of the generalized Cauchy-Fantappie formula on analytic subvarieties.

Remark 3. For fixed $z \in \tilde{D}$, we consider the following surface in $C^{2 n-2 m}$

$$
M_{z}=\left\{(\zeta, w): \sum_{j=1}^{n}\left(\zeta_{j}-z_{j}\right) w_{j}=1, \zeta \in \partial \tilde{D}\right\}
$$


We make the following assumptions: $w_{j}=w_{j}(\zeta, z),(\zeta, z) \in \partial \tilde{D} \times \tilde{D}, j=1, \cdots, n$, which belong to $C^{1}(\partial \tilde{D})$ in $\zeta$, and the condition $\sum_{j=1}^{n}\left(\zeta_{j}-z_{j}\right) w_{j}=1$ is fulfilled. $\tilde{C}_{0}$ denotes a cycle on $M_{z}$ and cycle $\widetilde{C}_{0}$ represents a homology class $\mathfrak{h} \in \mathscr{H}_{2 n-2 m-1}\left(M_{z}\right)$. Then, for any holomorphic function $f(z)$ in $\overline{\bar{D}}$ and any cycle $\tilde{C} \in \mathfrak{h}$, we have

$$
f(z)=c_{m} \int_{\tilde{C}} f(\zeta) \operatorname{det}_{(n)}\left(\frac{S_{0}}{T_{0}}, h_{1}, \cdots, h_{m}, d\left(\frac{S_{1}}{T_{1}}\right), \cdots, d\left(\frac{S_{n-m-1}}{T_{n-m-1}}\right)\right) \wedge B_{m}^{F}(\zeta) .
$$

Proof of theorem 2. According to the corollary of lemma 5, we have

$$
\begin{gathered}
\int_{\partial \tilde{D}} f(\zeta) \operatorname{det}_{(n)}\left(\frac{S_{0}}{T_{0}}, h_{1}, \cdots, h_{m}, \bar{\partial}_{\zeta}\left(\frac{S_{1}}{T_{1}}\right), \cdots, \bar{\partial}_{\zeta}\left(\frac{S_{n-m-1}}{T_{n-m-1}}\right)\right) \wedge B_{m}^{F}(\zeta) \\
=\int_{\partial \tilde{D}} f(\zeta) \operatorname{det}_{(n)}\left(\frac{S_{0}}{T_{0}}, h_{1}, \cdots, h_{m}, \bar{\partial}_{\zeta}\left(\frac{S_{0}}{T_{0}}\right), \cdots, \bar{\partial}_{\zeta}\left(\frac{S_{0}}{T_{0}}\right)\right) \wedge B_{m}^{F}(\zeta) \\
=\int_{\partial \tilde{D}} f(\zeta) \operatorname{det}_{(n)}\left(\frac{S_{0}}{T_{0}}, h_{1}, \cdots, h_{m}, \frac{\bar{\partial}_{\zeta} S_{0}}{T_{0}}, \cdots, \frac{\bar{\partial}_{\zeta} S_{0}}{T_{0}}\right) \wedge B_{m}^{F}(\zeta) \\
=\int_{\partial \tilde{D}} \frac{f(\zeta)}{T_{0}^{m-m}} \operatorname{det}_{(n)}\left(S_{0}, h_{1}, \cdots, h_{m}, \bar{\partial}_{\zeta} S_{0} \cdots, \bar{\partial}_{\zeta} S_{0}\right) \wedge B_{m}^{F}(\zeta)
\end{gathered}
$$

By further applying corollary 2 of lemma 3, we obtain (28).

Theorem 3. Let $D$ be a bounded domain with piecewise smooth boundaries in $\mathbb{C}^{n}$, the boundary $\partial D$ of $D$ consisting of a chain of slit spaces

$$
\partial D=\sigma^{(1)} \supset \cdots \supset \sigma^{(s)} \supset \sigma^{(t)} \supset \cdots \supset \sigma^{(\eta)} \supset \sigma^{(\beta)} .
$$

Assume that $\sigma^{(\beta)}$ be a $2 n-\beta$ dimensional boundary chain, i.e. there is a $2 n-\beta+1$ dimensional chain $\tau_{0}$, such that $\partial \tau_{0}=\sigma^{(\beta)}$. Correspondingly

$$
\partial \tilde{D}=\tilde{\sigma}^{(1)} \supset \cdots \supset \tilde{\sigma}^{(s)} \supset \tilde{\sigma}^{(t)} \supset \cdots \supset \tilde{\sigma}^{(\eta)} \supset \tilde{\sigma}^{(\beta)},
$$

$\partial \tau_{m}=\tilde{\sigma}^{(\beta)}$, and when $\zeta \in \partial \tilde{D},\left|\nabla_{m}^{F}(\zeta)\right| \neq 0$ and

$$
\operatorname{rank} \frac{\partial\left(N_{\beta}^{1}, \cdots, N_{\beta}^{n}\right)}{\partial\left(\bar{\zeta}_{1}, \cdots, \zeta_{n}\right)} \leqslant n-m-\beta
$$


Then, for a holomorphic function $f(z)$ on $\overline{\bar{D}}$ we have

$$
f(z)=c_{m} \int_{\tilde{\sigma}^{(\beta)} \times \Delta^{(\beta-1)}} f(\zeta) \operatorname{det}_{(n)}\left(Q, h_{1}, \cdots, h_{m}, \partial_{\bar{\zeta} \mu} Q, \cdots, \partial_{\bar{\zeta} \mu} Q\right) \wedge B_{m}^{F}(\zeta), \text { for } z \in \tilde{D}
$$

Proof. Let

$$
\begin{aligned}
& c=\partial\left(\tau_{0} \times \Delta^{(\beta-1)}\right)=\sigma^{(\beta)} \times \Delta^{(\beta-1)}+\varepsilon_{0} \tau_{0} \times \partial \Delta^{(\beta-1)}, \\
& \tilde{c}=\partial\left(\tau_{m} \times \Delta\left(^{\beta-1)}\right)=\tilde{\sigma}^{(\beta)} \times \Delta^{(\beta-1)}+\varepsilon \tau_{m} \times \partial \Delta^{(\beta-1)},\right.
\end{aligned}
$$

where $\varepsilon_{0}, \varepsilon= \pm 1$. Thus on $\Delta^{(\beta-1)}$, we have

$$
\begin{aligned}
\operatorname{det}_{(n)} & \left(Q, h_{1}, \cdots, h_{m}, \partial_{\zeta_{\mu}} Q, \cdots, \partial_{\bar{\zeta} \mu} Q\right) \\
& =\chi_{0}(\zeta, \mu)+\chi_{1}(\zeta, \mu)+\cdots+\chi_{\beta-1}(\zeta, \mu),
\end{aligned}
$$

where $\chi_{r}(\zeta, \mu)$ are differential forms, the degrees of $d \mu_{\theta}$ and $\overline{d \zeta}$ are $r$ and $n-m-r-1$ respectively. By $(31), \chi_{r}(\zeta, \mu)=0$, if $r<\beta-1$, and by the degree reasons, we have

$$
\int_{\tau_{m} \times \partial \Delta^{(\beta-1)}} f(\zeta) \chi_{\beta-1}(\zeta, \mu) \wedge B_{m}^{F}(\zeta)=0
$$

Thus

$$
\int_{\tau_{m} \times \partial \Delta^{(\beta-1)}} f(\zeta) \operatorname{det}_{(n)}\left(Q, h_{1}, \cdots, h_{m}, \partial_{\bar{\zeta} \mu} Q, \cdots, \partial_{\bar{\zeta} \mu} Q\right) \wedge B_{m}^{F}(\zeta)=0 .
$$

Therefore we obtain

$$
\begin{gathered}
\int_{\tilde{\sigma}^{(\beta)} \times \Delta^{(\beta-1)}} f(\zeta) \operatorname{det}_{(n)}\left(Q, h_{1}, \cdots, h_{m}, \partial_{\bar{\zeta} \eta} Q, \cdots, \partial_{\bar{\zeta}_{\eta}} Q\right) \wedge B_{m}^{F}(\zeta) \\
=\int_{\widetilde{C}} f(\zeta) \operatorname{det}_{(n)}\left(Q, h_{1}, \cdots, h_{m}, \partial_{\bar{\zeta}_{\mu}} Q, \cdots, \partial_{\bar{\zeta} \mu} Q\right) \wedge B_{m}^{F}(\zeta)
\end{gathered}
$$

On the other hand, let $\widetilde{C}_{1}=\partial\left(\tilde{D} \times \Delta_{0}^{(0)}\right)=\partial \tilde{D} \times \Delta_{0}^{(0)}$. Since $\tilde{C}$ and $\tilde{C}_{1}$ are the cycles of real dimension $2 n-2 m-1$, and 


$$
\begin{aligned}
& d\left[\operatorname{det}_{(n)}\left(Q, h_{1}, \cdots, h_{m}, \partial_{\bar{\zeta} \mu} Q, \cdots, \partial_{\bar{\zeta} \mu} Q\right) \wedge B_{m}^{F}(\zeta)\right] \\
& \quad=\operatorname{det}_{(n)}\left(\partial_{\bar{\zeta} \mu} Q, h_{1}, \cdots, h_{m}, \partial_{\bar{\zeta} \mu} Q, \cdots, \partial_{\bar{\zeta} \mu} Q\right) \wedge B_{m}^{F}(\zeta)=0
\end{aligned}
$$

on $\partial \tilde{D}=\tilde{\sigma}^{(1)}$, then

$$
\begin{aligned}
& \int_{C} f(\zeta) \operatorname{det}_{(n)}\left(Q, h_{1}, \cdots, h_{m}, \partial_{\bar{\zeta} \mu} Q, \cdots, \partial_{\bar{\zeta} \mu} Q\right) \wedge B_{m}^{F}(\zeta) \\
& \quad=\int_{C_{r}} f(\zeta) \operatorname{det}_{(n)}\left(Q, h_{1}, \cdots, h_{m}, \partial_{\bar{\zeta} \mu} Q, \cdots, \partial_{\bar{\zeta} \mu} Q\right) \wedge B_{m}^{F}(\zeta) \\
& =\int_{\partial \tilde{D} \times \Delta_{0}^{(0)}} f(\zeta) \operatorname{det}_{(n)}\left(Q, h_{1}, \cdots, h_{m}, \partial_{\bar{\zeta} \mu} Q, \cdots, \partial_{\bar{\zeta} \mu} Q\right) \wedge B_{m}^{F}(\zeta) \\
& =\int_{\partial \tilde{D}} \frac{f(\zeta)}{M_{0}^{(n-m)}} \operatorname{det}_{(n)}\left(N^{0}, h_{1}, \cdots, h_{m}, \partial_{\bar{\zeta}} N^{0}, \cdots, \partial_{\bar{\zeta}} N^{0}\right) \wedge B_{m}^{F}(\zeta) .
\end{aligned}
$$

Applying (34), (35) and (25), we obtain (32).

Theorem 4. Let $D$ be a nondegenerate polyhedral domain in $C^{n}$, such that its boundary $\partial D$ consists of a chain of slit spaces

$$
\partial D=\sigma_{1}^{(0)} \supset \sigma_{2}^{(0)} \supset \cdots \supset \sigma_{k}^{(0)} \supset \sigma_{k+1}^{(0)} \supset \sigma_{k+2}^{(0)} \supset \cdots \supset \sigma_{\beta}^{(0)},
$$

where $\sigma_{k+1}^{(0)}, \cdots, \sigma_{\beta}^{(0)}$ are the boundary surfaces of polyhedral type which is defined by pluriharmonic functions. Let $\Phi_{j_{v}}(\zeta, z)=\sum_{p=1}^{n}\left(\zeta_{p}-z_{p}\right) \varphi_{j_{v} p}(\zeta, z)$ be the holomorphic support functions on $\sigma_{k+1}^{(0)}$ and $H_{j_{v} p}=\varphi_{j_{v} p} / \Phi_{j_{v}}$. Let

$$
\partial \tilde{D}=\tilde{\sigma}_{1}^{(0)} \supset \tilde{\sigma}_{2}^{(0)} \supset \cdots \supset \tilde{\sigma}_{k}^{(0)} \supset \tilde{\sigma}_{k+1}^{(0)} \supset \tilde{\sigma}_{k+2}^{(0)} \supset \cdots \supset \tilde{\sigma}_{\beta}^{(0)}
$$

be the corresponding chain of slits of $\partial \tilde{D}$ and assume that $\left|\nabla_{m}^{F}(\zeta)\right| \neq 0$ on $\partial \tilde{D}$. Then for a holomorphic function $f(z)$ in $\overline{\bar{D}}$ we have

$$
\begin{gathered}
f(z)=\mathrm{c}_{m}^{0} \sum_{\substack{J_{\theta} \\
(k+1 \leqslant \theta \leqslant n-m)}} \int_{\tilde{\sigma}_{J_{\theta}}^{(0) \times \Delta_{J_{\theta-k}}}} f(\zeta) \sum_{\rho=1}^{k+1}(-1)^{k+1-\rho} \operatorname{det}_{(n)}\left(h_{1}, \cdots, h_{m}, H_{j_{1}}, \cdots\right. \\
\\
\left.\left[H_{j_{\rho}}\right], \cdots, H_{j_{k+1}}, Q, \partial_{\bar{\zeta} \mu} Q, \cdots, \partial_{\bar{\zeta} \mu} Q\right) \wedge B_{m}^{F}(\zeta), \text { for } z \in \tilde{D}
\end{gathered}
$$


where $C_{m}^{0}=(-1)^{m n}(-1)^{\frac{m(m+1)}{2}} / /^{(n-m) !(2 \pi i)^{n-m}}, J_{\theta}=\left\{j_{1}, \cdots, j_{\theta}\right\} \subset\{1, \cdots, N\}, j_{1}<\cdots<$ $J_{\theta} ; J_{\theta-k}=\left\{j_{1}, \cdots, j_{\theta-k}\right\} \subset\{1, \cdots, N\}, j_{1}<\cdots<j_{\theta-k}$.

Proof. It is easy to verify the following

$$
\begin{aligned}
& \partial\left(\sum_{\substack{J_{\theta} \\
(k+1 \leqslant \theta \leqslant N)}}(-1)^{\theta} \tilde{\sigma}_{J_{\theta}}^{(0)} \times \Delta_{0 J_{\theta-k}}\right)=\sum_{\substack{J_{\theta} \\
(k+1 \leqslant \theta \leqslant N)}} \tilde{\sigma}_{J_{\theta}}^{(\theta)} \times \Delta_{J_{\theta-k}}- \\
& \quad \sum_{j_{1}<\cdots<j_{k+1}} \tilde{\sigma}_{j_{1} \cdots j_{k+1}}^{(0)} \times \Delta_{0} .
\end{aligned}
$$

Since

$$
\left|\begin{array}{cccccccccc}
1 & 0 & \cdots & 0 & 1 & \cdots & 1 & 0 & \cdots & 0 \\
Q_{1} & h_{11} & \cdots & h_{m 1} & H_{j_{1} 1} & \cdots & H_{j_{k+1} 1} & \partial_{\bar{\zeta} \mu} Q_{1} & \cdots & \partial_{\bar{\zeta} \mu} Q_{1} \\
\vdots & \vdots & & \vdots & \vdots & & \vdots & \vdots & & \vdots \\
Q_{n} & h_{1 n} & \cdots & h_{m n} & H_{j_{1} n} & \cdots & H_{j_{k+1} n} & \partial_{\bar{\zeta} \mu} Q_{n} & \cdots & \partial_{\bar{\zeta} \mu} Q_{n}
\end{array}\right|=0
$$

on $\tilde{\sigma}_{J_{\theta}}^{(0)} \times \Delta_{0 J_{\theta-k}}(k+1 \leqslant \theta \leqslant N)$, then

$$
\begin{aligned}
& \sum_{\rho=1}^{k+1}(-1)^{k+1-\rho} \operatorname{det}_{(n)}\left(h_{1}, \cdots, h_{m}, H_{j_{1}}, \cdots,\left[H_{j_{\rho}}\right], \cdots, H_{j_{k+1}}, Q, \partial_{\bar{\zeta} \mu} Q, \cdots, \partial_{\bar{\zeta} \mu} Q\right) \\
& \quad=\operatorname{det}_{(n)}\left(h_{1}, \cdots, h_{m}, H_{j_{1}}, \cdots, H_{j_{k+1}}, \partial_{\bar{\zeta} \mu} Q, \cdots, \partial_{\bar{\zeta} \mu} Q\right) .
\end{aligned}
$$

Then

$$
\begin{aligned}
& d\left[\sum_{\rho=1}^{k+1}(-1)^{k+1-\rho} \operatorname{det}_{(n)}\left(h_{1}, \cdots, h_{m}, H_{j_{1}}, \cdots,\left[H_{j_{\rho}}\right], \cdots, H_{j_{k+1}}, Q, \partial_{\bar{\zeta} \mu} Q, \cdots, \partial_{\bar{\zeta} \mu} Q\right)\right. \\
& \left.\wedge B_{m}^{F}(\zeta)\right]=\partial_{\bar{\zeta} \mu} \operatorname{det}_{(n)}\left(h_{1}, \cdots, h_{m}, H_{j_{1}}, \cdots, H_{j_{k+1}}, \partial_{\bar{\zeta} \mu} Q, \cdots, \partial_{\bar{\zeta} \mu} Q\right) \wedge B_{m}^{F}(\zeta)=0 .
\end{aligned}
$$

It is from (37), (38) and Stokes' theorem, that

$$
\begin{aligned}
& C_{m}^{0} \quad \sum_{\substack{J_{\theta} \\
(k+1 \leqslant \theta \leqslant n-m)}} \int_{\widetilde{\sigma}_{J_{\theta}}^{(0)} \times \Delta_{J_{\theta-k}}} f(\zeta) \sum_{\rho=1}^{k+1}(-1)^{k+1-\rho} \operatorname{det}_{(n)}\left(h_{1}, \cdots, h_{m}, H_{j_{1}}, \cdots,\right. \\
& \left.\quad\left[H_{j_{\rho}}\right], \cdots, H_{j_{k+1}}, Q, \partial_{\bar{\zeta} \mu} Q, \cdots, \partial_{\bar{\zeta} \mu} Q\right) \wedge B_{m}^{F}(\zeta) \\
& =C_{m}^{0} \sum_{j_{1}<\cdots<j_{k+1}} \int_{\tilde{\sigma}_{j_{1} \cdots j_{k+1} \times \Delta_{0}}^{(0)}} f(\zeta) \sum_{\rho=1}^{k+1}(-1)^{k+1-\rho} \operatorname{det}_{(n)}\left(h_{1}, \cdots, h_{m}, H_{j_{1}}, \cdots,\right.
\end{aligned}
$$




$$
\begin{aligned}
& {\left.\left[\mathrm{H}_{j_{\rho}}\right], \cdots, H_{j_{k+1}}, Q, \partial_{\bar{\zeta} \mu} Q, \cdots, \partial_{\bar{\zeta} \mu} Q\right) \wedge B_{m}^{F}(\zeta) } \\
= & C_{m}^{0} \sum_{j_{1}<\cdots<j_{k+1}} \int_{\widetilde{\sigma}^{(0)} j_{1} \cdots j_{k+1}} \frac{f(\zeta)}{M_{0}^{n-m-1}} \sum_{\rho=1}^{k+1}(-1)^{k+1-\rho} \operatorname{det}_{(n)}\left(h_{1}, \cdots, h_{m}, H_{j_{1}}, \cdots,\right. \\
& {\left.\left[H_{j_{\rho}}\right], \cdots, H_{j_{k+1}}, N^{0}, \partial_{\bar{\zeta}} N^{0}, \cdots, \partial_{\bar{\zeta}} N^{0}\right) \wedge B_{m}^{F}(\zeta) . }
\end{aligned}
$$

Since

$$
\left|\begin{array}{cccccccccc}
1 & 0 & \cdots & 0 & 1 & \cdots & 1 & G & \cdots & G \\
\frac{N_{1}^{0}}{M_{0}} & h_{11} & \cdots & h_{m 1} & H_{j_{1} 1} & \cdots & H_{j_{k} 1} & \partial_{\bar{\zeta}} N_{1}^{0} & \cdots & \partial_{\bar{\zeta}} N_{1}^{0} \\
\vdots & \vdots & & \vdots & \vdots & & \vdots & \vdots & & \vdots \\
\frac{N_{n}^{0}}{M_{1}} & h_{1 n} & \cdots & h_{m n} & H_{j_{1} n} & \cdots & H_{j_{k} n} & \partial_{\bar{\zeta}} N_{n}^{0} & \cdots & \partial_{\bar{\zeta}} N_{n}^{0}
\end{array}\right|=0
$$

where $G=\sum_{j=1}^{n}\left(\zeta_{j}-z_{j}\right) \partial_{\bar{\zeta}} N_{j}^{0}$, then

$$
\begin{aligned}
& \frac{1}{\mathrm{M}_{0}^{n-m-k}} \operatorname{det}_{(n)}\left(h_{1}, \cdots, h_{m}, H_{j_{1}}, \cdots, h_{j_{k}}, \partial_{\bar{\zeta}} N^{0}, \cdots, \partial_{\bar{\zeta}} N^{0}\right)-\frac{n-m-k}{M^{n-m-k+1}} \\
& \quad G \wedge \operatorname{det}_{(n)}\left(h_{1}, \cdots, h_{m}, H_{j_{1}}, \cdots, H_{j_{k}}, N^{0}, \partial_{\bar{\zeta}} N^{0}, \cdots, \partial_{\bar{\zeta}} N^{0}\right) \\
& =\sum_{\rho=1}^{k}(-1)^{k-\rho} \operatorname{det}_{(n)}\left(h_{1}, \cdots, h_{m}, H_{j_{1}}, \cdots,\left[H_{j_{\rho}}\right], \cdots, H_{j_{k}},\right. \\
& \left.\quad \frac{N^{0}}{M_{0}^{n-m-k+1}}, \partial_{\bar{\zeta}} N^{0}, \cdots, \partial_{\bar{\zeta}} N^{0}\right) .
\end{aligned}
$$

On the other hand, we have

$$
\begin{aligned}
& \bar{\partial}_{\zeta} \operatorname{det}_{(n)}\left(h_{1}, \cdots, h_{m}, H_{j_{1}}, \cdots,\left[H_{j_{\rho}}\right], \cdots, H_{j_{k+1}}, \frac{N^{0}}{M_{0}^{n-m-k}}, \partial_{\bar{\zeta}} N^{0}, \cdots, \partial_{\bar{\zeta}} N^{0}\right) \\
= & \operatorname{det}_{(n)}\left(h_{1}, \cdots, h_{m}, H_{j_{1}}, \cdots,\left[H_{j_{\rho}}\right], \cdots, H_{j_{k+1}}, \bar{\partial}_{\zeta}\left(\frac{N^{0}}{M_{0}^{n-m-k}}\right), \partial_{\bar{\zeta}} N^{0}, \cdots, \partial_{\bar{\zeta}} N^{0}\right) \\
= & \frac{1}{M_{0}^{n-m-k}} \operatorname{det}_{(n)}\left(h_{1}, \cdots, h_{m}, H_{j_{1}}, \cdots,\left[H_{j_{\rho}}\right], \cdots, H_{j_{k+1}}, \partial_{\bar{\zeta}} N^{0}, \cdots, \partial_{\bar{\zeta}} N^{0}\right)
\end{aligned}
$$




$$
=\frac{n-m-k}{M_{0}^{n-m-k+1}} G \wedge \operatorname{det}_{(n)}\left(h_{1}, \cdots, h_{m}, H_{j_{1}}, \cdots,\left[H_{j_{\rho}}\right], \cdots, H_{j_{k+1}}, N^{0}, \partial_{\bar{\zeta}} N^{0}, \cdots, \partial_{\bar{\zeta}} N^{0}\right)
$$

on $\partial \tilde{D}$.

$$
\begin{aligned}
& \text { Moreover, since } \partial \tilde{\sigma}_{j_{1} \cdots\left[j_{\rho}\right] \cdots j_{k+1}}^{(0)}=\bigcup_{j_{\rho}}(-1)^{k+1-\rho} \tilde{\sigma}_{j_{1} \cdots j_{k+1}}^{(0)} \text {, and } \\
& \sum_{j_{1}<\cdots<j_{k+1}}=\frac{1}{(k+1) !} \sum_{j_{1}, \cdots, j_{k+1}},
\end{aligned}
$$

then by Stokes' formula and (41), (42), we obtain

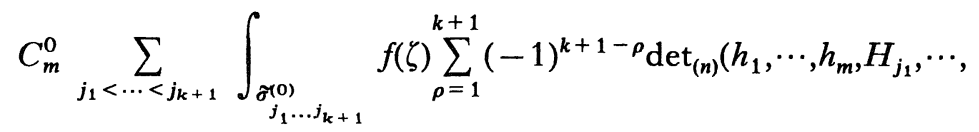

$$
\begin{aligned}
& \left.\left[H_{j_{\rho}}\right], \cdots, H_{j_{k+1}}, \frac{N^{0}}{M_{0}^{n-m-k}}, \partial_{\bar{\zeta}} N^{0}, \cdots, \partial_{\bar{\zeta}} N^{0}\right) \wedge B_{n}^{F}(\zeta) \\
& =C_{m}^{0} \frac{1}{(k+1) !} \sum_{\rho=1}^{k+1} \sum_{j_{1}, \cdots,\left[j_{\rho}\right], \cdots, j_{k+1}} \sum_{j_{\rho}} \int_{\substack{\widetilde{\sigma}_{j_{1}}^{(0)}, j_{k+1} \\
j_{1}, 1}}(-1)^{k+1-\rho} f(\zeta) \operatorname{det}_{(n)}\left(h_{1},\right. \\
& \left.\cdots, h_{m}, H_{j_{1}}, \cdots,\left[H_{j_{\rho}}\right], \cdots, H_{j_{k+1}}, \frac{N^{0}}{M_{0}^{n-m-k}}, \partial_{\bar{\zeta}} N^{0}, \cdots, \partial_{\bar{\zeta}} N^{0}\right) \wedge B_{m}^{F}(\zeta)
\end{aligned}
$$

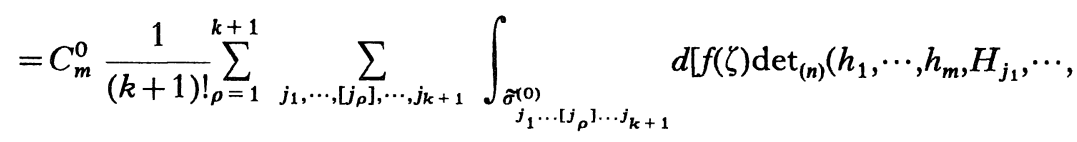

$$
\begin{aligned}
& \left.\left.\left[H_{j_{\rho}}\right], \cdots, H_{j_{k+1}}, \frac{N^{0}}{M_{0}^{n-m-k}}, \partial_{\bar{\zeta}} N^{0}, \cdots, \partial_{\bar{\zeta}} N^{0}\right) \wedge B_{m}^{F}(\zeta)\right] \\
& =C_{m}^{0} \frac{1}{k !} \sum_{j_{1}, \cdots, j_{k}} \int_{\substack{\tilde{\sigma}_{j_{1} \cdots j_{k}}^{(0)} \\
M_{1}}} f(\zeta)\left[\frac { 1 } { M _ { 0 } ^ { n - m - k } } \operatorname { d e t } _ { ( n ) } \left(h_{1}, \cdots, h_{m}, H_{j_{1}}, \cdots, H_{j_{k}},\right.\right. \\
& \left.\partial_{\bar{\zeta}} N^{0}, \cdots, \partial_{\bar{\zeta}} N^{0}\right) \wedge B_{m}^{F}(\zeta)-\frac{n-m-k}{M_{0}^{n-m-k+1}} G \wedge \operatorname{det}_{(n)}\left(h_{1}, \cdots, h_{m}, H_{j_{1}}, \cdots\right. \\
& \left.H_{j_{k}}, N^{0}, \partial_{\bar{\zeta}} N^{0}, \cdots, \partial_{\bar{\zeta}} N^{0}\right) \wedge B_{m}^{F}(\zeta)
\end{aligned}
$$




$$
\begin{aligned}
= & C_{m}^{0} \sum_{j_{1}<\cdots<j_{k}} \int_{\substack{\tilde{\sigma}_{j_{1} \cdots j_{k}}^{(0)}\\
}} f(\zeta) \sum_{\rho=1}^{k}(-1)^{k-\rho} \operatorname{det}_{(n)}\left(h_{1}, \cdots, h_{m}, H_{j_{1}} \cdots,\left[H_{j_{\rho}}\right], \cdots, H_{j_{k}},\right. \\
& \left.\frac{N^{0}}{M_{0}^{n-m-k+1}}, \partial_{\bar{\zeta}} N^{0}, \cdots, \partial_{\bar{\zeta}} N^{0}, \cdots, \partial_{\bar{\zeta}} N^{0}\right) \wedge B_{m}^{F}(\zeta)
\end{aligned}
$$

Using (43) repeatedly we obtain

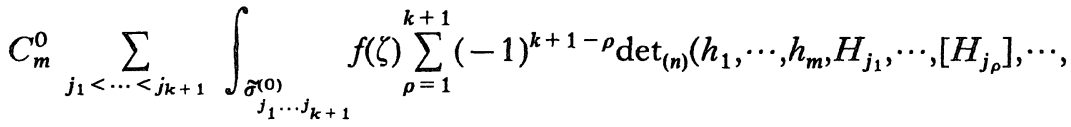

$$
\begin{aligned}
& \left.H_{j_{k+1}}, \frac{N^{0}}{M_{0}^{n-m-k}}, \partial_{\bar{\zeta}} N^{0}, \cdots, \partial_{\bar{\zeta}} N^{0}\right) \wedge B_{m}^{F}(\zeta) \\
& =C_{m}^{0} \sum_{j_{1}} \int_{\substack{\widetilde{\sigma}_{j_{1}}^{(0)} \\
\text { (n) }}} f(\zeta) \operatorname{det}_{(n)}\left(h_{1}, \cdots, h_{m}, \frac{N^{0}}{M_{0}^{n-m},}, \partial_{\bar{\zeta}} N^{0}, \cdots, \partial_{\bar{\zeta}} N^{0}\right) \wedge B_{m}^{F}(\zeta) \\
& =C_{m} \int_{\partial \tilde{D}} \frac{f(\zeta)}{M_{0}^{n-m}} \operatorname{det}_{(n)}\left(N^{0}, h_{1}, \cdots, h_{m}, \partial_{\bar{\zeta}} N^{0}, \cdots, \partial_{\bar{\zeta}} N^{0}\right) \wedge B_{m}^{F}(\zeta) .
\end{aligned}
$$

Applying (25) to the right-hand side of (44), we have

$$
\begin{gathered}
f(z)=C_{m}^{0} \sum_{j_{1}<\cdots<j_{k+1}} \int_{\substack{\widetilde{\sigma}_{j_{1} \cdots j_{k+1}}^{(0)} \\
M_{0}^{n-m-k}}} \frac{f(\zeta)}{\sum_{\rho=1}^{k+1}}(-1)^{k+1-\rho} \operatorname{det}_{(n)}\left(h_{1}, \cdots, h_{m},\right. \\
\\
\left.H_{j_{1}}, \cdots,\left[H_{j_{\rho}}\right], \cdots, h_{j_{k+1}}, N^{0}, \partial_{\bar{\zeta}} N^{0}, \cdots, \partial_{\bar{\zeta}} N^{0}\right) \wedge B_{m}^{F}(\zeta) .
\end{gathered}
$$

(39) and (45) imply (36).

Remark. When $k=n-m-1$, (45) can be rewritten as:

$$
\begin{aligned}
f(z)= & C_{m}^{0} \sum_{j_{1}<\cdots<j_{n-m}} \int_{\substack{\widetilde{\sigma}_{j_{1} \cdots j_{n-m}}^{(0)} \\
M_{0}}} \frac{f(\zeta)}{\rho=1} \sum_{\rho=1}^{n-m}(-1)^{n-m-\rho} \operatorname{det}_{(n)}\left(h_{1}, \cdots, h_{m}, H_{j_{1}}, \cdots\right. \\
& {\left.\left[H_{j_{\rho}}\right], \cdots, H_{j_{n-m}}, N^{0}\right) \wedge B_{m}^{F}(\zeta) } \\
= & C_{m}^{0} \sum_{j_{1}<\cdots<j_{n-m}} \int_{\substack{\widetilde{\sigma}_{j_{1} \cdots j_{n-m}}^{(0)}\\
}} f(\zeta) \operatorname{det}_{(n)}\left(h_{1}, \cdots, h_{m}\right. \\
& \left.H_{j_{1}}, \cdots, H_{j_{n-m}}\right), \wedge B_{m}^{F}(\zeta)
\end{aligned}
$$


Let $D$ be a holomorphic polyhedron. Then (45) and (46) are generalizations $^{[8]}$ of the integral representation formulas of holomorphic functions for analytic polyhedrons ${ }^{[4]}$ in analytic subvarieties (the generalization of Weil's integral representation in analytic subvarieties ${ }^{[7]}$ is also included).

\section{References}

[1] Henkin, G.M., Integral representation of functions in strictly pseudoconvex domains and applications to the $\bar{\partial}$-problem, Math. sb., 82 (1970), 300-309.

[2] Ramirex de Arellano, E., Ein Divisionsproblem und Randintegraldarstellungen in der komplexen Analysis, Math. Ann., 184 (1970), 172-187.

[3] Range, R.M. and Siu, Y.T., Uniform estimates for the $\bar{\partial}$-equation on domains with piecewise smooth strictly pseudoconvex boundaries, Math. Ann., 206 (1973), 325-354.

[4] Sommer, F., Über die Integralformein in der Funktionentheorie mehrerer Komplexer Veräuderlicher, Math. Ann., 125 (1952), 172-182.

[ 5 ] Sergeev, A.G. and Henkin, G.M., Uniform estimates for the $\bar{\partial}$-equation on pseudoconvex polyhedron, Math. Sb., 112 (154) (1980), 522-567.

[6] Stout, E.L., An integral formula for holomorphic functions on strictly pseudoconvex hypersurfaces, Duke Math. J., 42 (1975), 347-356.

[ 7 ] Hatziafratis, T.E., Integral representation formulas on analytic varieties, Pacific J. Math., 123 (1986), 71-91.

[ 8 ] Chen Shu-jin, The Integral representation of the holomorphic functions on the analytic family, Acta Sci. Natur. Univ. Amoien, 29 (1990), 1-6. (in Chinese).

[ 9 ] Look, C.H., On Cauchy-Fantappie formula, Acta Math. Sinica, 16 (1966), 344-363. (in Chinese) 
